\title{
Polyallylamine hydrochloride coating enhances the fluorescence emission of Human Serum Albumin encapsulated gold nanoclusters
}

\author{
Ben Allan Russell ${ }^{a}+$ Barbara Jachimska $^{\mathrm{b}}$ and Yu Chen ${ }^{\mathrm{a}}$
}

a. Department of Physics, University of Strathclyde, Glasgow, G4 ONG, United Kingdom

b. Jerzy Haber Institute of Catalysis and Surface Chemistry Polish Academy of Sciences, Krakow, PL30329, Poland

† Corresponding Author E.mail: ben.russell@strath.ac.uk.

\section{Abstract}

Protein encapsulated gold nanoclusters have received much attention due to the possibility of using them as a non-toxic fluorescent probe or marker for biomedical applications, however one major disadvantage currently is their low brightness and quantum yield in comparison to currently used fluorescent markers. A method of increasing the fluorescence emission of Human Serum Albumin (HSA) encapsulated gold nanoclusters (AuNCs) via a Polyallylamide hydrochloride (PAH) coating is described. PAH molecules with a molecular weight of $\sim 17,500$ Da were found to enhance the fluorescence emission of HSA-AuNCs by 3-fold when the protein/polymer concentration ratio is 2:1 in solution. Interestingly, the fluorescence lifetime of the AuNCs was found to decrease while the native tryptophan (TRP) fluorescence lifetime also decreased during the fluorescence emission intensity enhancement caused by the PAH binding. Coinciding with the decrease in fluorescence lifetime, the zeta potential of the system was observed to be zero during maximum fluorescence intensity enhancement, causing the formation of large aggregates. These results suggest that PAH binds to the HSA-AuNCs acting as a linker; causing aggregation and rigidification, which results in a decrease in separation between native TRP of HSA and AuNCs; improving Förster Resonance Energy Transfer (FRET) and increasing the fluorescence emission intensity. These findings are critical to the development of brighter protein encapsulated AuNCs.

\section{Keywords}

Fluorescent Gold Nanoclusters; Protein Encapsulated Gold Nanoclusters; Fluorescence Enhancement; Forster Resonance Energy Transfer; Fluorescence Lifetime; Fluorophore

\section{Introduction}

Several studies have been undertaken to understand the unique fluorescent properties of protein encapsulated gold nanoclusters (AuNCs)[1-3]. Their red band emission peak, non-toxicity and non-photobleaching nature make them perfectly suited for biological imaging and sensing[4,5]. However, in their current state protein encapsulated AuNCs have very low brightness levels, with quantum yields reported at $~ 6 \%[6]$. Of all the protein encapsulated AuNCs which have been synthesised, human serum albumin (HSA) AuNCs have received the most 
attention[7-11]. Serum albumin is the most abundant protein found in mammalian blood, acting as a transportation molecule for a variety of molecules including drugs, thus much research has been undertaken to fully understand the protein characteristics in different environments and conditions[12-17]. Previous research on the native protein offers insights as to why HSA encapsulated AuNC fluorescence characteristics change in differing environments. Previously it has been shown that the fluorescence emission of HSA-AuNCs is quenched in low $\mathrm{pH}$ solutions due to the exposure of AuNCs to the solvent as the HSA protein unfolds[18]. The physicochemical characteristics of HSA-AuNCs has also been studied to understand the effects AuNCs synthesis has on the proteins natural characteristics[19]. Of major interest was the large shift in the protein's surface charge to a highly negative charge state at neutral $\mathrm{pH}$ values and lends itself to the possibility that positively charged molecules can be bound to the surface; allowing for the modification of the HSA-AuNCs, enhancing the fluorescence emission brightness. Fan et. al. successfully enhanced the fluorescence emission brightness of $\mathrm{CdSeS/ZnS}$ quantum dots by 2 orders of magnitude via a PMMA-co-MMA polymer[20]. To explore these enhancements a positively charged polymer, polyallylamine hydrochloride (PAH), was chosen as a means of coating due to the negative surface charge of the HSA-AuNC complex. PAH is typically used as a means of creating a highly hydrophobic film in conjunction with polysodium styrene sulfonate (PSS), forming a layer by layer negatively and positively charged film, which is typically used in microencapsulation of molecules and nanoparticles[21,22].

\section{Materials and Methods}

HSA-AuNCs were synthesised using the commonly cited one-pot method developed by Xie et. al.[23]. $17 \mathrm{mg}$ of $\mathrm{HAuCl}_{4}$ and $250 \mathrm{mg}$ of $\mathrm{HSA}$ were dissolved separately in two beakers with $5 \mathrm{ml}$ of water at $37^{\circ} \mathrm{C}$. Once dissolved the two solutions were mixed together for 2 mins. Next $0.5 \mathrm{ml}$ of $\mathrm{NaOH}$ at a concentration of $1 \mathrm{M}$ was added to the mixture, increasing the $\mathrm{pH}$ above 10 . The solution was then stirred and kept at a constant $37^{\circ} \mathrm{C}$ for 6 hours. After 6 hours the sample was then kept at $37{ }^{\circ} \mathrm{C}$ in an oven for $24 \mathrm{hrs}$. The concentrated solution was then purified after synthesis using $10 \mathrm{kDa}$ dialysis cassettes. Dialysis was carried out in a PBS buffer solution $(\mathrm{I}=0.01$ $\mathrm{M}, \mathrm{pH}=7.4)$. The dialysed HSA-AuNCs solution was diluted to $40 \mu \mathrm{M}$ with PBS buffer solution $(\mathrm{I}=0.01 \mathrm{M}, \mathrm{pH}=$ 7.4) for use during all fluorescence based experiments. A stock solution of PAH ( $M w=17,500 \mathrm{Da}$ ) was prepared at a concentration of $1 \mathrm{mM}$ for use in all fluorescence based experiments. For Zeta potential and Dynamic Light Scattering (DLS) experiments, the concentration of HSA-AuNCs and PAH stock concentration were diluted by $37.5 \%$ to $15.0 \mu \mathrm{M}$ and $375 \mu \mathrm{M}$, respectively. All chemicals were purchased from Sigma Aldrich. Fluorescence emission data was collected using a Horiba Fluorolog 3. Fluorescence lifetime data was collected using a Horiba Deltaflex. All experiments carried out on the Horiba Deltaflex were carried out using a NanoLED pulsed light source (excitation wavelength $295 \mathrm{~nm}$ ). A Time-to-amplitude converter (TAC) range of $100 \mathrm{~ns}$ was used to study tryptophan (TRP) fluorescence lifetimes, a TAC range of $13 \mu$ s was used for AuNCs fluorescence lifetimes. Dynamic Light Scattering (DLS) and electrophoretic mobility experiments were carried out on a Malvern Zetasizer NS. UV-Vis measurements were carried out on a Thermofisher Evolution 201 Spectrophotometer.

\section{Results and Discussion}

To illustrate the maximum fluorescence enhancement of HSA-AuNCs after adding a polymer coating the emission spectra for native HSA-AuNCs and HSA-AuNCs with PAH added (HSA-AuNCs-PAH) excited at $290 \mathrm{~nm}$ and $470 \mathrm{~nm}$ are shown in Figure 1. 


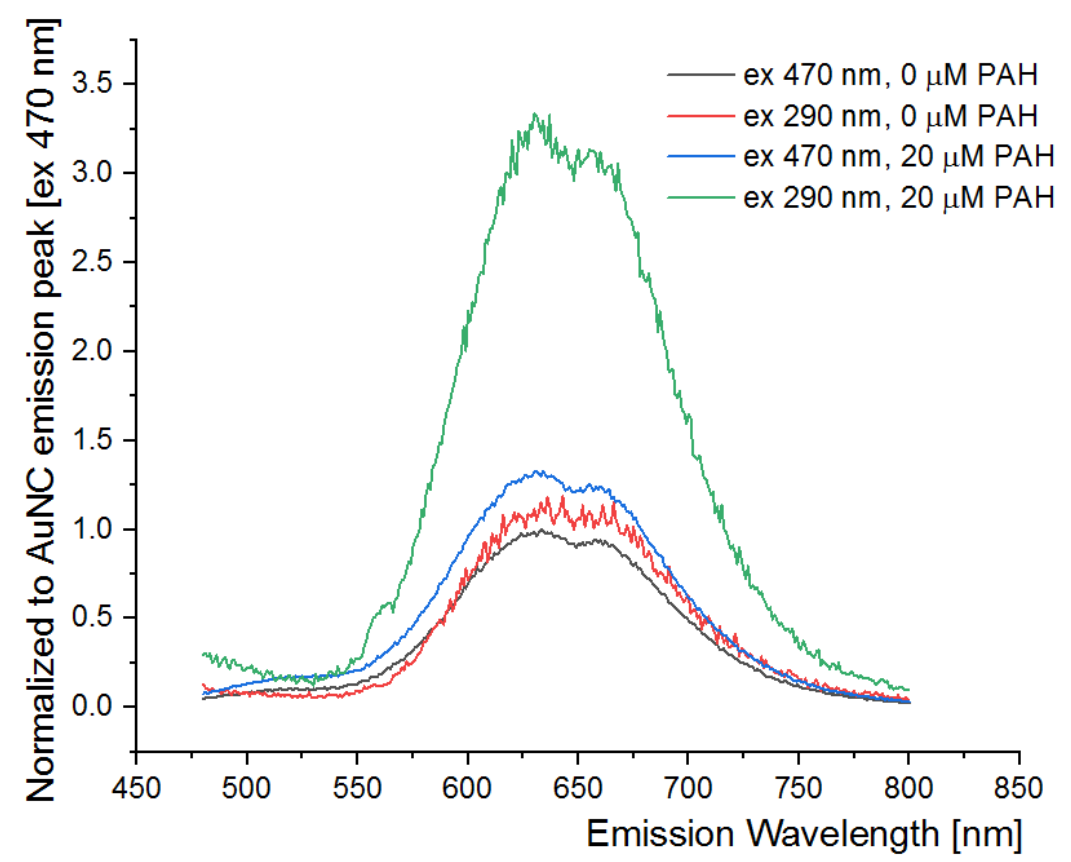

Figure 1. Fluorescence emission spectra of HSA-AuNCs without PAH coating and HSA-AuNCS with 20 $\mu \mathrm{M}$ PAH coating. Excitation wavelength of $290 \mathrm{~nm}$ and $470 \mathrm{~nm}$.

The fluorescence spectrum of HSA-AuNCs after the $20 \mu \mathrm{M}$ PAH coating when exciting at $290 \mathrm{~nm}$ can be seen to increase 3 -fold while the emission peak position is slightly redshifted by $8 \mathrm{~nm}$. When directly exciting the AuNC using $470 \mathrm{~nm}$ excitation a fluorescence enhancement is also evident however it is not as substantial as exciting at $290 \mathrm{~nm}$ where the AuNC is excited predominately via a Forster Resonance Energy Transfer (FRET) from the single TRP of HSA to the AuNC $[9,18]$. To find the best ratio of PAH:HSA-AuNCs, small additions of PAH were made to a solution of HSA-AuNCs and the maximum emission intensity for both excitation at $290 \mathrm{~nm}$ and $470 \mathrm{~nm}$ were measured, as shown in Figure 2.

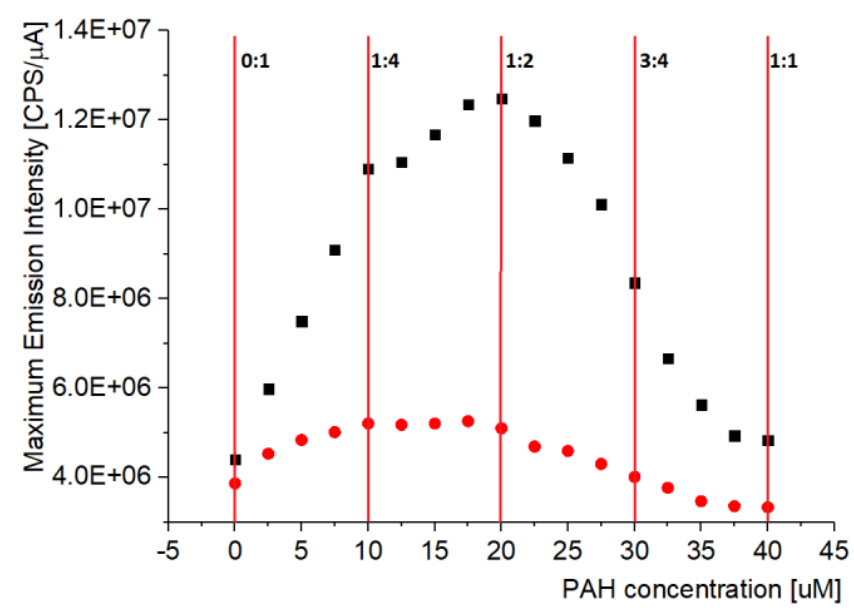

Figure 2. Maximum emission intensity of HSA-AuNCs as a function of added PAH concentration. Peak emission intensity for $290 \mathrm{~nm}$ excitation (black), $470 \mathrm{~nm}$ (red). HSA-AuNCs concentration is kept at a concentration of $40.0 \mu \mathrm{M}$ throughout. Vertical red lines indicate the molecular ratio between PAH:HSA-AuNCs.

From Figure 2 we can see that the addition of PAH coating to HSA-AuNCs results in a gradual increase in fluorescence emission from the AuNCs until a maximum is reached at a concentration of $20 \mu \mathrm{M}$ of PAH and it returns to the original fluorescence intensity at $40 \mu \mathrm{M}$. The fluorescence enhancement when exciting at $290 \mathrm{~nm}$ 
can be explained by an improvement in the FRET process due to the coating of the HSA-AuNC with PAH causing a change in conformation that may bring the TRP and AuNC closer together enhancing the FRET[24-27]. However, no FRET takes place at $470 \mathrm{~nm}$ excitation and a different enhancement mechanism must be responsible for the increase in intensity observed. A decrease in collisional quenching due to the PAH shielding the HSA-AuNC complex from solution may account for fluorescence intensity enhancement. Another possibility is the AuNC is rigidified by the PAH interaction, causing an increase in emission via the $A u(I)$ shell[28]. Increasing the PAH concentration from $40 \mu \mathrm{M}$ to $200 \mu \mathrm{M}$ resulted in no further changes to the fluorescence emission intensity when exciting at $290 \mathrm{~nm}$ and $470 \mathrm{~nm}$. Changes to the peak emission wavelength were also observed upon the addition of PAH to a HSA-AuNC solution, as shown in Figure 3.

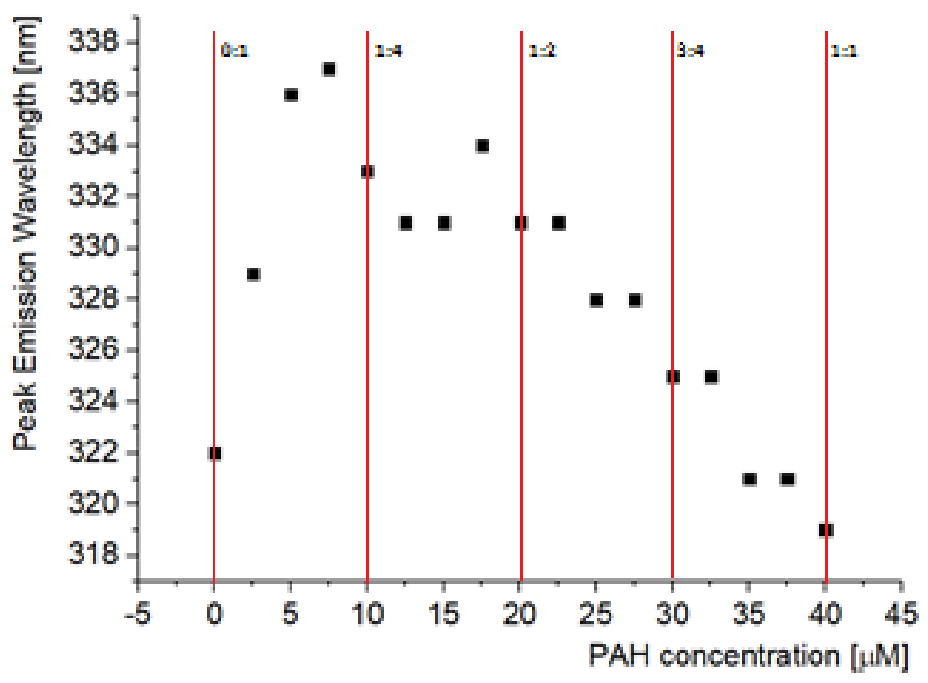

Figure 3. Peak emission wavelength of tryptophan fluorescence from HSA-AuNCs as a function of PAH concentration in solution. Excitation wavelength $290 \mathrm{~nm}$.

A red shift in the peak emission wavelength of $10 \mathrm{~nm}$ when excited at $290 \mathrm{~nm}$ and a red shift of $5 \mathrm{~nm}$ when excited at $470 \mathrm{~nm}$ was observed. The red shift may be explained by changes in the TRP microenvironment brought about by PAH interactions. The red shift of TRP results in a reduced spectral overlap between TRP emission and AuNC absorption. Therefore, improved FRET must arise from a reduced donor-acceptor pair separation. The maximum emission intensity of TRP was also monitored while adding PAH, shown in Figure 4.

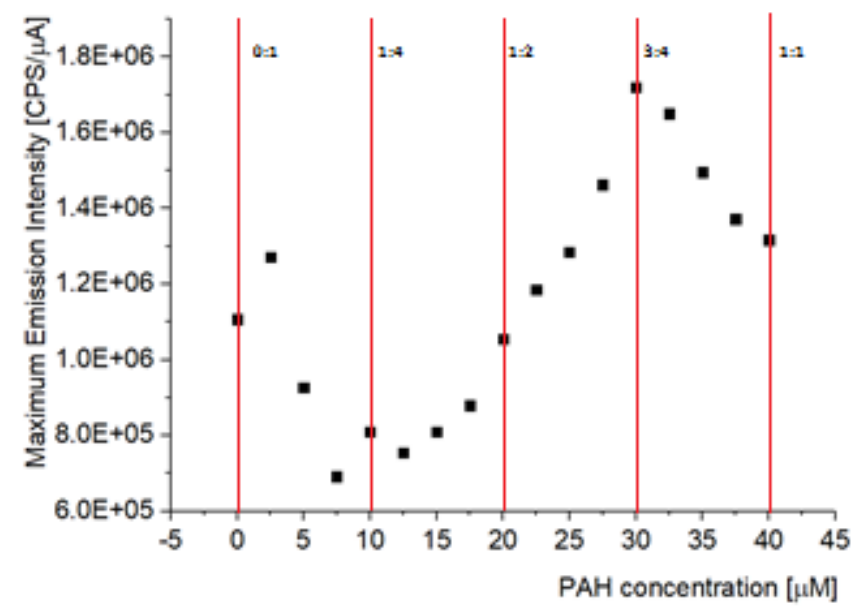

Figure 4. Maximum emission intensity of tryptophan fluorescence from HSA-AuNCs as a function of PAH concentration in solution. Excitation wavelength $290 \mathrm{~nm}$. 
The maximum fluorescence intensity of TRP was observed to decrease (as AuNC emission increased), as a result of increased PAH concentration. This decrease in TRP fluorescence intensity is an indicator of increased FRET efficiency. It is possible that the decrease is also linked to a decrease in quantum yield of TRP due to the presence of the positively charged PAH nearby affecting the electron transfer between the TRP indole ring and acceptor during fluorescence[29]. However, a decrease in donor quantum yield would result in a decrease in FRET efficiency. Therefore the improvement in FRET efficiency observed here may be dominated by the decrease in the donor-acceptor separation. Fluorescence lifetime measurements of the AuNC and TRP were both taken and 3 exponential fittings were used to describe best the fluorescence lifetime decays measured for TRP and AuNC emission as previous studies [18,30]. The fluorescence decay of AuNCs consists of two $\mu$ s duration fluorescence lifetimes arising from AuNC emission and one shorter lifetime resulting from scattered light. The fluorescence decay of TRP consists of three ns duration fluorescence lifetimes arising from 3 different rotamer forms of the TRP residue, as shown in Table 1.

Table 1. Fluorescence lifetimes and relative amplitudes of the fluorescence decays associated of TRP and AuNCS with different concentrations of PAH added.

\begin{tabular}{|c|c|c|c|c|}
\hline $\begin{array}{c}\text { Emission. Source } \\
\text { (PAH concentration) }\end{array}$ & $\begin{array}{c}\tau_{1} \mathrm{~ns} \\
\text { (rel. amp) }\end{array}$ & $\begin{array}{c}\tau_{2} \mathrm{~ns} \\
\text { (rel. amp) }\end{array}$ & $\begin{array}{c}\tau_{3} \mathrm{~ns} \\
\text { (rel. amp) }\end{array}$ & $\chi^{2}$ \\
\hline TRP $(0 \mu \mathrm{M}$ PAH $)$ & $\begin{array}{l}1.05 \pm 0.05 \\
(47.13 \%)\end{array}$ & $\begin{array}{c}2.89 \pm 0.51 \\
(4.90 \%)\end{array}$ & $\begin{array}{l}5.78 \pm 0.20 \\
(17.98 \%)\end{array}$ & 1.45 \\
\hline TRP $(20 \mu \mathrm{M}$ PAH $)$ & $\begin{array}{l}0.08 \pm 0.01 \\
(85.10 \%)\end{array}$ & $\begin{array}{c}1.55 \pm 0.27 \\
(8.17 \%)\end{array}$ & $\begin{array}{c}3.75 \pm 0.33 \\
(6.73 \%)\end{array}$ & 1.48 \\
\hline TRP (40 $\mu \mathrm{M}$ PAH) & $\begin{array}{l}0.27 \pm 0.07 \\
(99.86 \%)\end{array}$ & $\begin{array}{c}1.52 \pm 0.02 \\
(0.11 \%)\end{array}$ & $\begin{array}{c}4.27 \pm 0.30 \\
(0.03 \%)\end{array}$ & 1.71 \\
\hline AuNC $(0 \mu \mathrm{M}$ PAH $)$ & $\begin{array}{l}100 \pm 30 \\
(7.01 \%)\end{array}$ & $\begin{array}{l}1020 \pm 140 \\
(26.17 \%)\end{array}$ & $\begin{array}{l}2220 \pm 40 \\
(66.82 \%)\end{array}$ & 1.73 \\
\hline AuNC (20 $\mu \mathrm{M}$ PAH) & $\begin{array}{l}20 \pm 20 \\
(2.00 \%)\end{array}$ & $\begin{array}{l}130 \pm 80 \\
(6.01 \%)\end{array}$ & $\begin{array}{l}1430 \pm 240 \\
(92.99 \%)\end{array}$ & 1.37 \\
\hline AuNC (40 $\mu \mathrm{M}$ PAH) & $\begin{array}{l}60 \pm 40 \\
(7.66 \%)\end{array}$ & $\begin{array}{l}1040 \pm 290 \\
(30.79 \%)\end{array}$ & $\begin{array}{l}2250 \pm 90 \\
(61.55 \%)\end{array}$ & 1.51 \\
\hline
\end{tabular}

The high $\chi^{2}$ values of the fittings are possibility due to the high amount of light scattering experienced due to AuNCs and particle aggregation. The initial fluorescence lifetimes of 1.02 and $2.22 \mu$ s observed before adding $\mathrm{PAH}$ to the HSA-AuNC solution were consistent with previous studies[18]. Upon adding $20 \mu \mathrm{M}$ of PAH, the fluorescence lifetimes of AuNCs was seen change dramatically, with one lifetime of $1.43 \mu$ secoming the dominant fluorescence source with a relative amplitude of $92.99 \%$. The other shorter fluorescence lifetimes are attributed to scattered light. The decrease in fluorescence lifetime while intensity increases indicate that the fluorescence enhancement is not caused by reduced collisional quenching and is more likely due to the rigidification mechanism previously reported[28]. A decreasing lifetime in conjunction with an increasing fluorescence intensity was also previously observed when adding native Lysozyme to Ly-AuNCs, which was attributed to aggregation and changes to the complexes' conformation[31]. Upon increasing the PAH concentration to $40 \mu \mathrm{M}$, the fluorescence lifetimes were seen to return to their previous state. Before adding $\mathrm{PAH}$ to the HSA-AuNC solution, the TRP fluorescence has 3 fluorescence lifetimes. Upon adding $20 \mu \mathrm{M}$ of PAH the lifetimes become shorter; the two longer lifetimes decrease to 1.55 and $3.75 \mathrm{~ns}$, with the shorter lifetime of 0.08 ns becoming dominant with a relative amplitude of $85.10 \%$. Upon increasing the PAH concentration further, the shorter fluorescence lifetime becomes even more dominant with $99.86 \%$, however the values of the 2 longer fluorescence lifetimes do not change. When TRP becomes exposed to water, its fluorescence lifetime decay curve has been shown to change from having 3 exponential fluorescence dynamics to 2 exponential fluorescence dynamics; with the 2 lifetimes being the same duration as the 2 longer fluorescence lifetimes observed[32]. The changes to the fluorescence lifetime of TRP may indicate a change in the protein conformation upon PAH interaction. The third shorter lifetime seen when adding PAH can be explained by the PAH coating increasing the particle size of HSA-AuNCs and thus more effectively scattering light. This is supported by the shorter fluorescence lifetime's relative amplitude increasing upon the addition of further $\mathrm{PAH}$. To further understand the physical changes which PAH addition induces to HSA-AuNCs, the particle size and zeta potential of the HSA-AuNC solution while adding PAH was determined. The particle size of HSA-AuNCs were seen 
reach a peak size when the HSA-AuNC fluorescence emission is at a maximum and then decrease with a further increase in the PAH concentration to the solution, as shown in Figure 5.

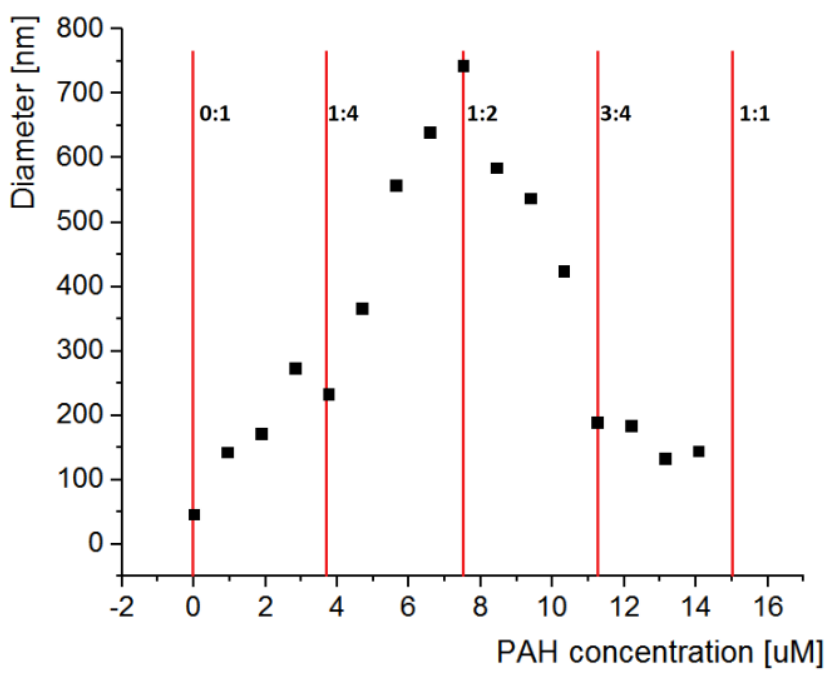

Figure 5. The diameter of HSA-AuNCS/PAH complexes as a function of PAH concentration in solution. HSAAuNCs concentration is kept at a concentration of $7.50 \mu \mathrm{M}$ throughout. Vertical red lines indicate the molecular ratio between PAH:HSA-AUNCS.

Interestingly, the largest complex sizes were observed when the overall zeta potential of the solution complex was near zero, as shown in Figure 6.

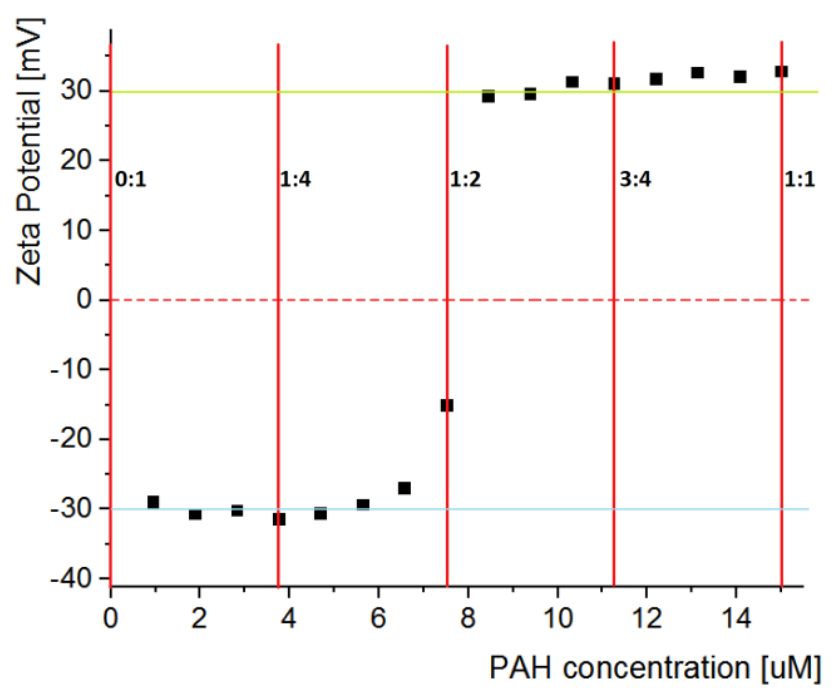

Figure 6. The zeta potential of HSA-AuNCS/PAH complexes as a function of PAH concentration in solution. HSAAuNCs concentration is kept at a concentration of $7.5 \mu \mathrm{M}$ throughout. Vertical red lines indicate the molecular ratio between PAH:HSA-AuNCs. Green horizontal line represents $P A H$ zeta potential at $p H 7.4^{22}$. Blue vertical line represents HSA-AUNC zeta potential at $\mathrm{pH} 7.4^{12}$.

From the diameter and zeta potential results we can clearly see that the enhancement of fluorescence emission from HSA-AuNCs coincides with an increase in the complex aggregate diameter which results from a lack of electrostatic repulsion between the HSA-AuNCs/PAH in solution, since the overall net charge of the solution at a 1:2 molecular ratio is close to zero. Previously we observed that a solution of HSA-AuNCs at pH 7.4 has a zeta potential of $-30 \mathrm{mV}[19]$. The zeta potential of $\mathrm{PAH}$ at $\mathrm{pH} 7.4$ was previously observed to be $30 \mathrm{mV}$ due to partial 
protonation (70 \%)[33], with the isoelectric point for $\mathrm{PAH}$ being $\mathrm{pH}$ 10.7[34]. As the overall zeta potential becomes positive the HSA-AuNC/PAH complex aggregation size becomes smaller and the fluorescence emission enhancement decreases. It is possible that the PAH molecule acts as a linker, joining HSA-AuNC molecules together as previously observed for BSA/PAH interactions[35]. As the PAH concentration is increased, more HSAAuNCs aggregate together until the ratio is at 1:2 PAH:HSA-AuNCs. Upon adding further PAH, more HSA-AuNC molecules have the linker and are unable to aggregate due to electrostatic repulsion of the PAH molecule. A change in the overall surface charge due to increased concentration of PAH molecules may also cause stiffening of the HSA-AuNC/PAH aggregates formed at larger PAH concentration. Previously, a blue shift in AuNC fluorescence emission was observed when doping silica nanoparticles with bovine serum albumin encapsulated AuNCs[36], as seen when the Zeta potential becomes positive in this system upon adding PAH at a ratio higher than 1:2 to the HSA-AuNC complex. One explanation attributed to the blue shift was the increased stiffness of the microenvironment inside the silica nanoparticle where the BSA-AuNCs were seen to reside.

\section{Conclusions}

To conclude, we have found a new method of increasing the brightness of the fluorescence emission from HSAAuNCs using a PAH polymer coating. HSA-AuNCs coated in PAH at a molecule ratio of 1:2 resulted in an increase of AuNC fluorescence emission intensity of 2.5 times when excited at $290 \mathrm{~nm}$, a smaller increase was observed when exciting the AuNC directly at $470 \mathrm{~nm}$. We believe the increase in fluorescence intensity may be due to either an enhancement of FRET between TRP and AuNCs when excited at $290 \mathrm{~nm}$ due to a decrease in donor acceptor separation and rigidification of the HSA-AuNC by the PAH acting as a linker between HSA-AuNC molecules, causing aggregation. The increase in fluorescence intensity when exciting directly at $470 \mathrm{~nm}$ arises from only the PAH causing aggregation and rigidification of the AuNC. Upon further addition of PAH, the fluorescence enhancement was diminished and fluorescence emission returned to normal levels. This may be attributed to the reduced aggregation and rigidification resulting from the repulsion between HSA-AuNC/PAH molecules as higher ratios of PAH:HSA-AuNC result in increased positive surface charges. It is hoped that this study will advance the development of new strategies of improving the brightness of other protein encapsulated AuNCs based on either rigidifying the protein/AuNC complex or protecting the complex from the solvent environment, which will support the adoption of this new type of fluorophore in biological sensing and imaging applications.

\section{Acknowledgements}

This work was partially supported by National Science Centre (NCN); OPUS 2016/23/B/ST5/02788.

\section{References}

[1] Y. Xu, J. Sherwood, Y. Qin, D. Crowley, M. Bonizzoni, Y. Bao, The role of protein characteristics in the formation and fluorescence of Au nanoclusters, Nanoscale. 6 (2014) 1515-1524. doi:10.1039/c3nr06040c.

[2] S. Raut, R. Chib, R. Rich, D. Shumilov, Z. Gryczynski, I. Gryczynski, Polarization properties of fluorescent BSA protected Au25 nanoclusters., Nanoscale. 5 (2013) 3441-3446. doi:10.1039/c3nr34152f.

[3] X.-L.L. Cao, H.-W.W. Li, Y. Yue, Y. Wu, pH-Induced conformational changes of BSA in fluorescent AuNCs@BSA and its effects on NCs emission, Vib. Spectrosc. 65 (2013) 186-192. doi:10.1016/j.vibspec.2013.01.004. 
[4] L. Shang, G.U. Nienhaus, Gold nanoclusters as novel optical probes for in vitro and in vivo fluorescence imaging, Biophys. Rev. 4 (2012) 313-322. doi:10.1007/s12551-012-0076-9.

[5] L.Y. Chen, C.W. Wang, Z. Yuan, H.T. Chang, Fluorescent gold nanoclusters: Recent advances in sensing and imaging, Anal. Chem. 87 (2015) 216-229. doi:10.1021/ac503636j.

[6] X. Qu, Y. Li, L. Li, Y. Wang, J. Liang, J. Liang, Fluorescent gold nanoclusters: Synthesis and recent biological application, J. Nanomater. 2015 (2015). doi:10.1155/2015/784097.

[7] B.A. Russell, P.A. Mulheran, D.J.S. Birch, Y. Chen, Probing the Sudlow binding site with warfarin: How does gold nanocluster growth alter human serum albumin?, Phys. Chem. Chem. Phys. 18 (2016). doi:10.1039/c6cp03428d.

[8] P.-H. Chan, Y.-C. Chen, Human serum albumin stabilized gold nanoclusters as selective luminescent probes for Staphylococcus aureus and methicillin-resistant Staphylococcus aureus., Anal. Chem. 84 (2012) 8952-6. doi:10.1021/ac302417k.

[9] S. Raut, R. Chib, S. Butler, J. Borejdo, Z. Gryczynski, I. Gryczynski, Evidence of energy transfer from tryptophan to BSA/HSA protected gold nanoclusters, Methods Appl. Fluoresc. 2 (2014) 035004. doi:10.1088/2050-6120/2/3/035004.

[10] M. Santhosh, S.R. Chinnadayyala, A. Kakoti, P. Goswami, Selective and sensitive detection of free bilirubin in blood serum using human serum albumin stabilized gold nanoclusters as fluorometric and colorimetric probe., Biosens. Bioelectron. 59 (2014) 370-6. doi:10.1016/j.bios.2014.04.003.

[11] B.A. Russell, A. Garton, A.S. Alshammari, D.J.S. Birch, Y. Chen, Sudlow site II of human serum albumin remains functional after gold nanocluster encapsulation: a fluorescence-based drug binding study of L-Dopa, Methods Appl. Fluoresc. 6 (2018) 035017. doi:10.1088/20506120/aacdee.

[12] B.X. Huang, H.-Y. Kim, C. Dass, Probing three-dimensional structure of bovine serum albumin by chemical cross-linking and mass spectrometry., J. Am. Soc. Mass Spectrom. 15 (2004) 1237-47. doi:10.1016/j.jasms.2004.05.004.

[13] M.L. Ferrer, R. Duchowicz, B. Carrasco, J.G. de la Torre, A.U. Acuña, The conformation of serum albumin in solution: a combined phosphorescence depolarization-hydrodynamic modeling study., Biophys. J. 80 (2001) 2422-30. doi:10.1016/S0006-3495(01)76211-X.

[14] K. Tokarczyk, K. Kubiak-Ossowska, B. Jachimska, P.A. Mulheran, The Energy Landscape of Negatively Charged BSA Adsorbed on a Negatively Charged Silica Surface, J. Phys. Chem. B. (2018). doi:10.1021/acs.jpcb.7b12484.

[15] A.K. Shaw, S.K. Pal, Spectroscopic studies on the effect of temperature on $\mathrm{pH}$-induced folded states of human serum albumin, J. Photochem. Photobiol. B Biol. 90 (2008) 69-77. doi:10.1016/j.jphotobiol.2007.11.003.

[16] O.J. Rolinski, A. Martin, D.J.S. Birch, Human serum albumin and quercetin interactions monitored by time-resolved fluorescence: evidence for enhanced discrete rotamer conformations., J. Biomed. Opt. 12 (2008) 034013. doi:10.1117/1.2747623.

[17] K. Kubiak-Ossowska, K. Tokarczyk, B. Jachimska, P.A. Mulheran, Bovine Serum Albumin Adsorption at a Silica Surface Explored by Simulation and Experiment, J. Phys. Chem. B. 121 (2017) 3975-3986. doi:10.1021/acs.jpcb.7b01637.

[18] B.A. Russell, K. Kubiak-Ossowska, P.A. Mulheran, D.J.S. Birch, Y. Chen, Locating the Nucleation Sites for Protein Encapsulated Gold Nanoclusters: A Molecular Dynamics and Fluorescence 
Study, Phys. Chem. Chem. Phys. 17 (2015) 21935-21941. doi:10.1039/C5CP02380G.

[19] B.A. Russell, B. Jachimska, I. Kralka, P.A. Mulheran, Y. Chen, Human serum albumin encapsulated gold nanoclusters: effects of cluster synthesis on natural protein characteristics, J. Mater. Chem. B. 4 (2016) 6876-6882. doi:10.1039/C6TB01827K.

[20] Y. Fan, H. Liu, R. Han, L. Huang, H. Shi, Y. Sha, Y. Jiang, Extremely high brightness from polymer-encapsulated quantum dots for two-photon cellular and deep-tissue imaging., Sci. Rep. 5 (2015) 9908. doi:10.1038/srep09908.

[21] R. Luo, S.S. Venkatraman, B. Neu, Layer-by-Layer Polyelectrolyte-Polyester Hybrid Microcapsules for Encapsulation and Delivery of Hydrophobic Drugs, Biomacromolecules. 14 (2013) 2262-2271. doi:10.1021/bm4003915.

[22] Y. Zhang, D. Xu, W. Li, J. Yu, Y. Chen, Effect of size, shape, and surface modification on cytotoxicity of gold nanoparticles to human HEp-2 and Canine MDCK Cells, J. Nanomater. 2012 (2012). doi:10.1155/2012/375496.

[23] J. Xie, Y. Zheng, J.Y. Ying, Protein-directed synthesis of highly fluorescent gold nanoclusters, J. Am. Chem. Soc. 131 (2009) 888-889. doi:10.1021/ja806804u.

[24] D. Kajihara, R. Abe, I. lijima, C. Komiyama, M. Sisido, T. Hohsaka, FRET analysis of protein conformational change through position-specific incorporation of fluorescent amino acids, Nat. Methods. 3 (2006) 923-929. doi:10.1038/nmeth945.

[25] P. Chanphai, L. Bekale, S. Sanyakamdhorn, D. Agudelo, H.A. Tajmir-Riahi, Effect of synthetic polymers on polymer-protein interaction, Polym. (United Kingdom). 55 (2014) 572-582. doi:10.1016/j.polymer.2013.12.026.

[26] H.-X. Zhou, Polymer Crowders and Protein Crowders Act Similarly on Protein Folding Stability, FEBS Lett. 587 (2013) 394-397. doi:10.1016/j.febslet.2013.01.030.

[27] B. Farruggia, G. García, C. D’Angelo, G. Picó, Destabilization of human serum albumin by polyethylene glycols studied by thermodynamical equilibrium and kinetic approaches, Int. J. Biol. Macromol. 20 (1997) 43-51. doi:10.1016/S0141-8130(96)01150-6.

[28] K. Pyo, V.D. Thanthirige, K. Kwak, P. Pandurangan, G. Ramakrishna, D. Lee, Ultrabright Luminescence from Gold Nanoclusters: Rigidifying the Au(I)-Thiolate Shell, J. Am. Chem. Soc. 137 (2015) 8244-8250. doi:10.1021/jacs.5b04210.

[29] P.R. Callis, T. Liu, Quantitative Prediction of Fluorescence Quantum Yields for Tryptophan in Proteins, J. Phys. Chem. B. 108 (2004) 4248-4259. doi:10.1021/jp0310551.

[30] M. Amiri, K. Jankeje, J.R. Albani, Origin of Fluorescence Lifetimes in Human Serum Albumin. Studies on Native and Denatured Protein, J. Fluoresc. 20 (2010) 651-656. doi:10.1007/s10895-010-0597-1.

[31] N. Alkudaisi, B.A. Russell, D.J.S. Birch, Y. Chen, Gold Nanocluster Fluorescence Lifetime Sensitivity to Early Stage Lysozyme Aggregation Under Acidic Conditions, Submiss. (2018).

[32] J.T. Vivian, P.R. Callis, Mechanisms of Tryptophan Fluorescence Shifts in Proteins, Biophys. J. 80 (2001) 2093-2109. doi:http://dx.doi.org/10.1016/S0006-3495(01)76183-8.

[33] B. Jachimska, T. Jasiński, P. Warszyński, Z. Adamczyk, Conformations of poly(allylamine hydrochloride) in electrolyte solutions: Experimental measurements and theoretical modeling, Colloids Surfaces A Physicochem. Eng. Asp. 355 (2010) 7-15. doi:https://doi.org/10.1016/j.colsurfa.2009.11.012. 
[34] J. Choi, M.F. Rubner, Influence of the Degree of Ionization on Weak Polyelectrolyte Multilayer Assembly, Macromolecules. 38 (2005) 116-124. doi:10.1021/ma048596o.

[35] V. Ball, M. Winterhalter, P. Schwinte, P. Lavalle, J.C. Voegel, P. Schaaf, Complexation mechanism of bovine serum albumin and poly(allylamine hydrochloride), J. Phys. Chem. B. 106 (2002) 2357-2364. doi:10.1021/jp012522m.

[36] X. Le Guevel, B. Hotzer, G. Jung, M. Schneider, NIR-emitting fluorescent gold nanoclusters doped in silica nanoparticles, J. Mater. Chem. 21 (2011) 2974-2981. doi:10.1039/COJM02660C.

\section{Highlights}

- HSA-AuNCs coated with a PAH polymer ( $\mathrm{mW} 17,500 \mathrm{Da}$ ) in different concentrations.

- 2.5 fold enhancement of fluorescence emission of HSA-AuNCs induced by PAH binding..

- Optimum ratio for fluorescence enhancement between HSA-AuNC/PAH is 2:1.

- Excitation pathway via $290 \mathrm{~nm}$ excitation more affected by PAH binding than that via $470 \mathrm{~nm}$ excitation.

- Enhancement is likely due to improved FRET efficiency between TRP and AuNCs, and rigidification of the HSA-AuNC complex.

\section{Graphical Abstract}

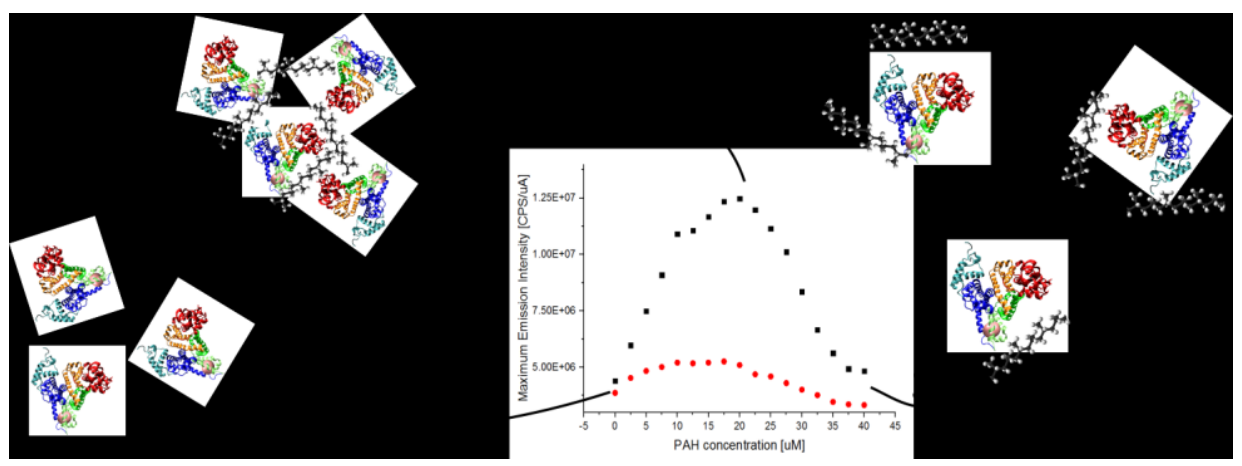

